\title{
The Study of Air Flow for Uniform Profile Numerical Simulation and Influence Factors
}

\author{
XinLei Li
}

School of energy,power and mechanical engineering,North China Electric Power University,Baoding 071000, China

lixinlei24@163.com

\begin{abstract}
This article use Fluent to make numerical simulation of air flow for uniform and around airfoil profile. By changing different angle of attacks and different profile shapes, I analyze the distribution of pressure v velocity and temperature field around profile to further evident the application value of boundary layer theory.
\end{abstract}

Keywords: Airfoil profile, boundary layer, flow around, numerical simulation

\section{Introduction}

Plant put forward boundary layer theory in 1904. It said that the viscous effect ranks only second to the thin layer closed to the object surface while the influence of thin layer external viscous can be ignored. It has great importance across the time. Not only opened up the new prospect of viscous fluid mechanics applying to practical problems, but further clarifying the study of perfect fluid practical significance.

This article use the method of numerical simulation to study air flow for uniform and around profile distribution of the flow, analyzing different angle of attacks and different profile shapes in pressure 、 velocity and temperature field. ${ }^{[1]}$ Boundary layer was applied during the analysis procedure and I deeply understand the significant of boundary layer theory.

\section{Numerical Simulation}

\subsection{Condition Setting of Grid and Boundary}

\section{a. Mesh Generation}

Models in this article has 27360 grids in total. Here are grid charts and relevant settings:
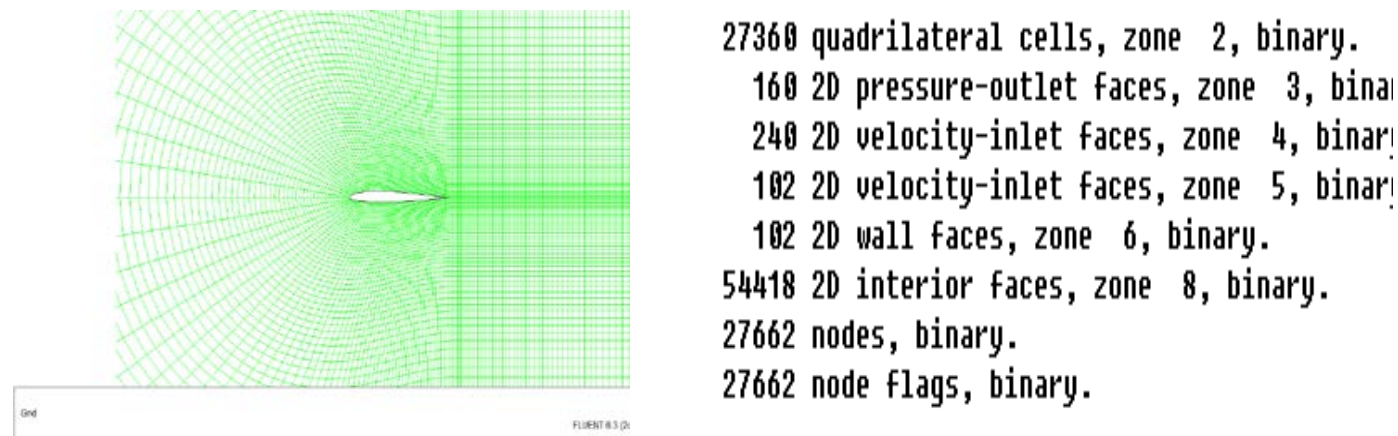

FIGURE 1. Grid charts and relevant settings

\section{b.The Settings of Boundary Layer}

Fluent adopts planar double precision, setting the fluid to the air and using model. The density of the air is $1.225 \mathrm{~kg} / \mathrm{m}^{3}$, the temperature is $300 \mathrm{~K}$, the viscosity is $1.7894 \mathrm{e}-05 \mathrm{~kg} /\left(\mathrm{m}^{*} \mathrm{~s}\right)$. The above parameters are for a fixed value and the airfoil chord length is $1 \mathrm{~m}$ approximately. To avoid inlet and outlet boundary effects profile flow, ${ }^{[2]}$ We choose profile ahead radius is $10 \mathrm{~m}$, and rear area is $20 * 20$ square. Residual error sets $10^{-6}$. During the study of Reynolds number and angle of attack, the profile adopts EN1.0. 
2.2 Flow Field Analysis in Different Angle of Attacks

a. Distribution of Pressure
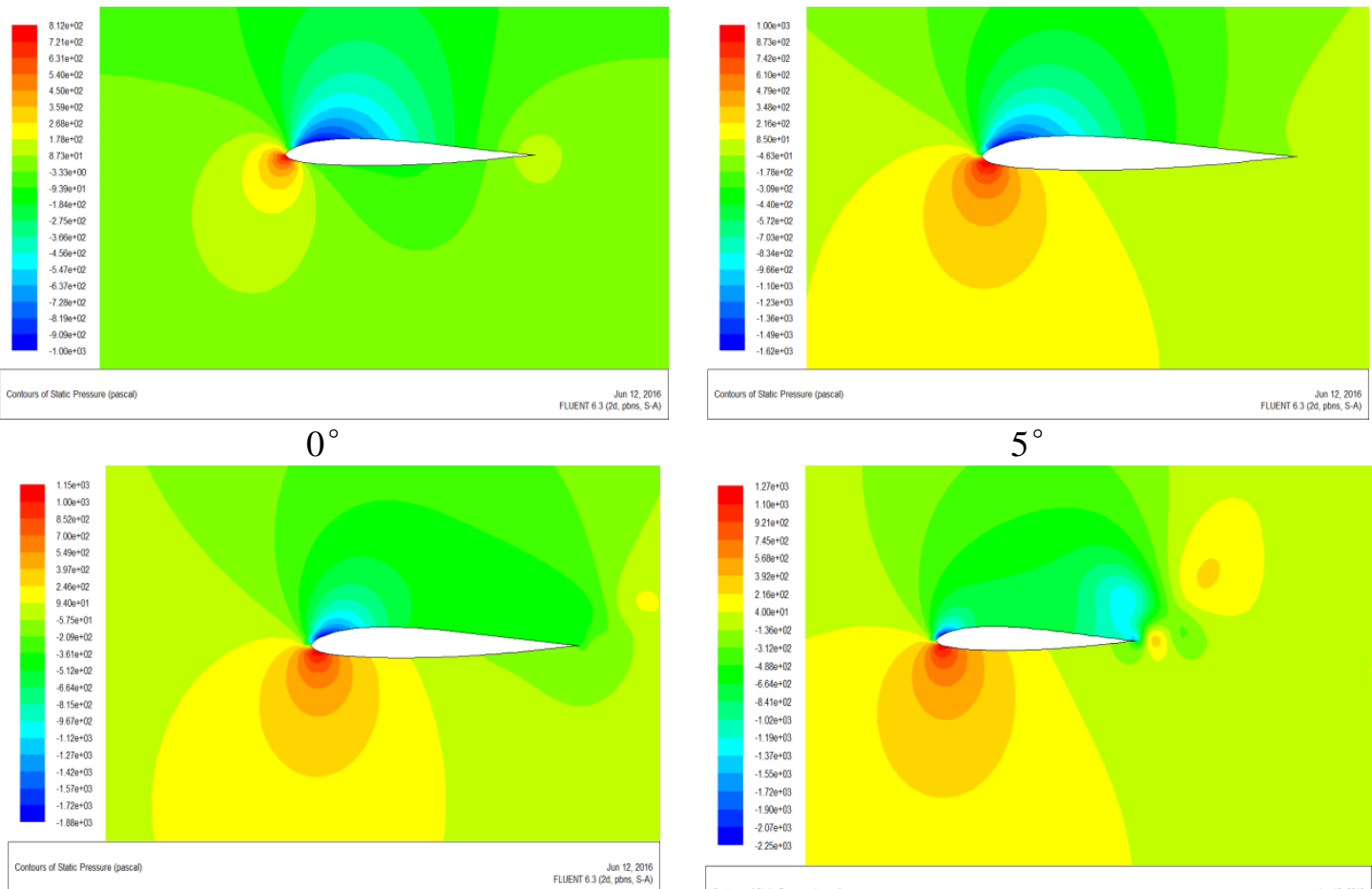

$10^{\circ}$
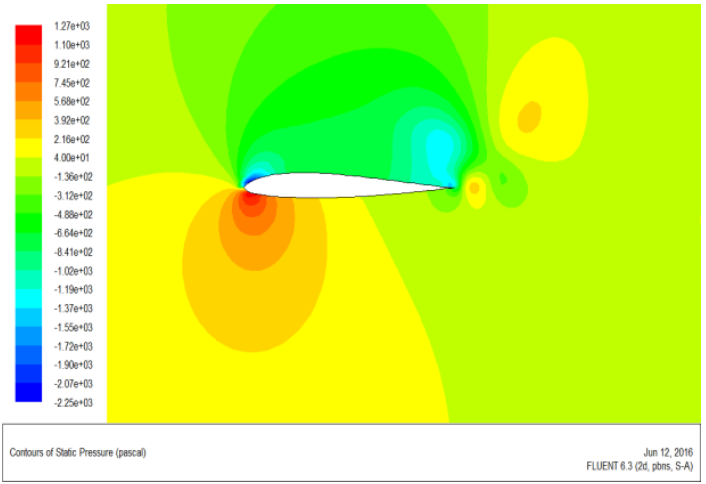

$15^{\circ}$

FIGURE 2. Pressure distribution of different angle of attacks

b. Velocity Vector
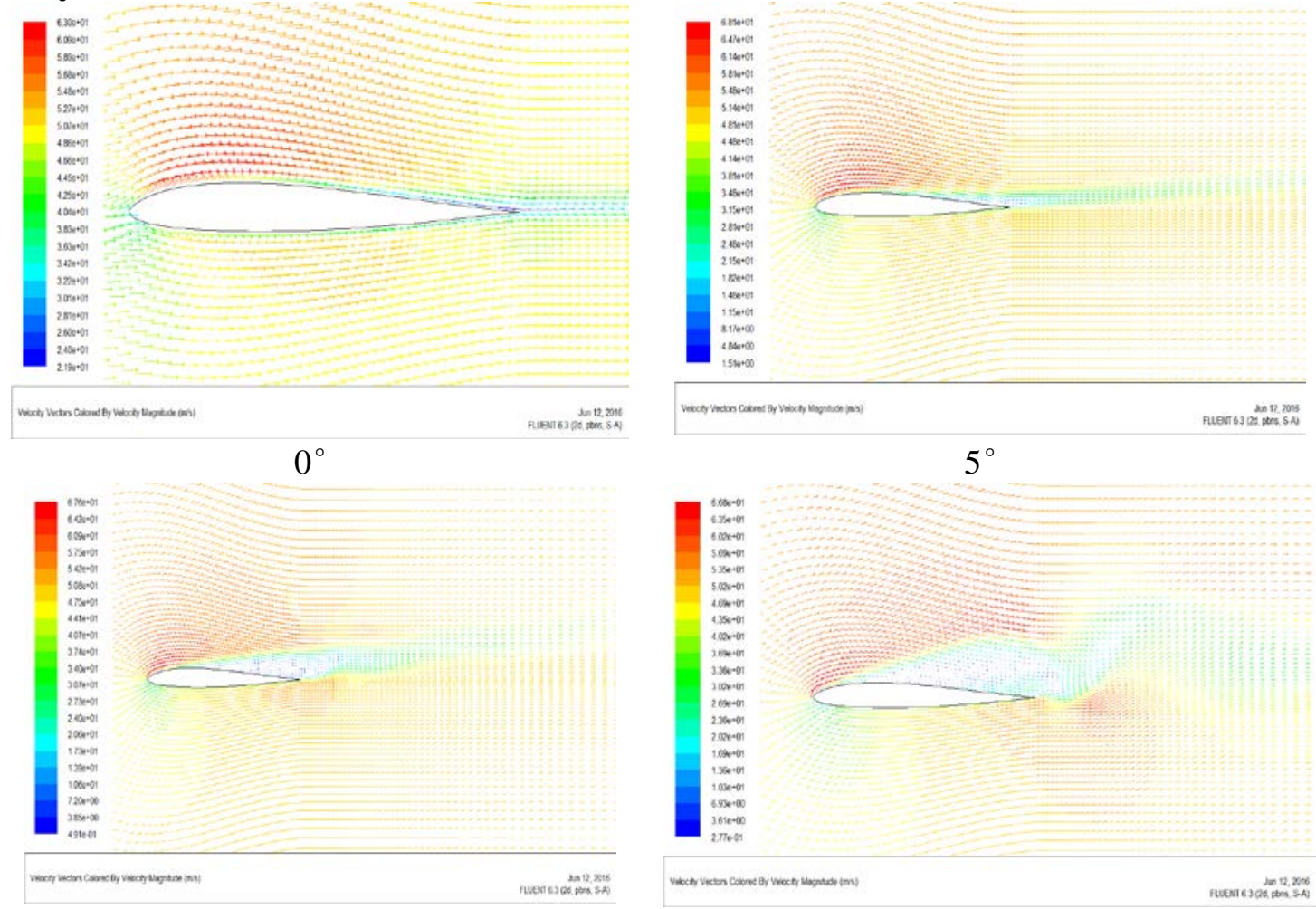

$15^{\circ}$

FIGURE 3. Velocity distribution of different angle of attacks 


\subsection{Flow field analysis in different profile shapes}

\section{a. Distribution of Pressure}

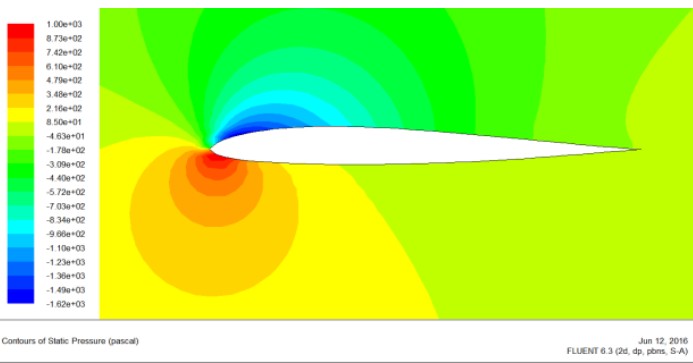

EN1.0

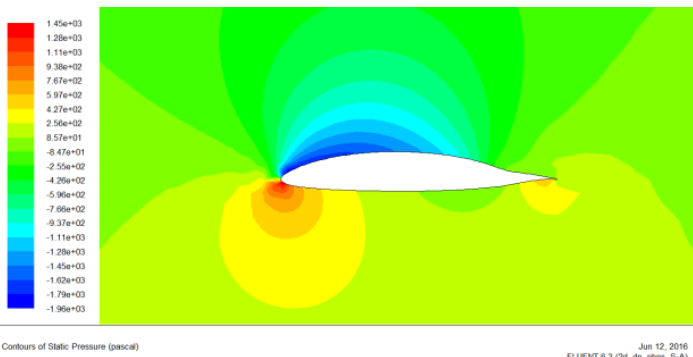

AH79K143

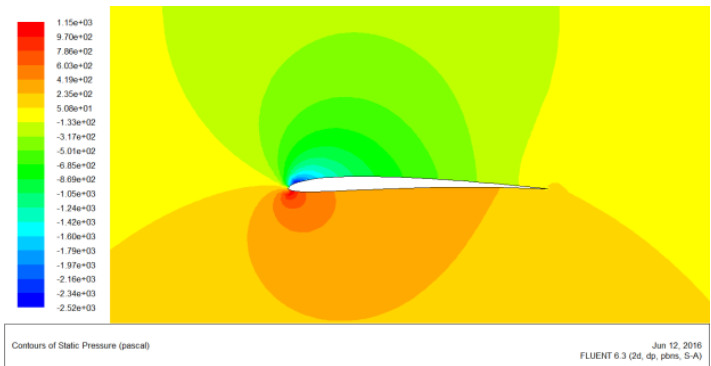

AG19

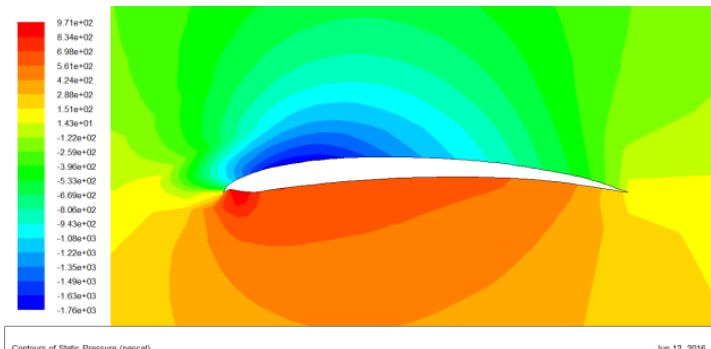

AH7476

FIGURE 4. Distribution of pressure in different profile shapes

\section{b. Distribution of Velocity}

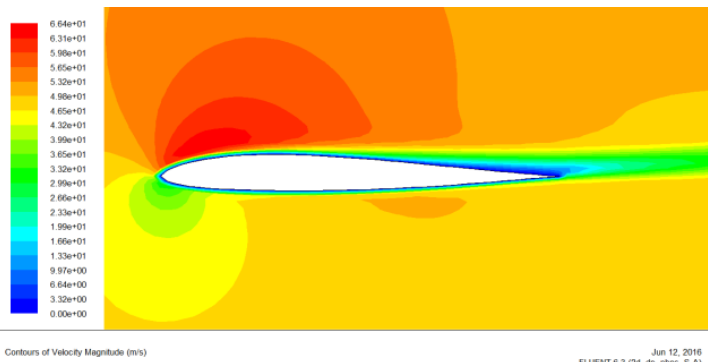

EN1.0

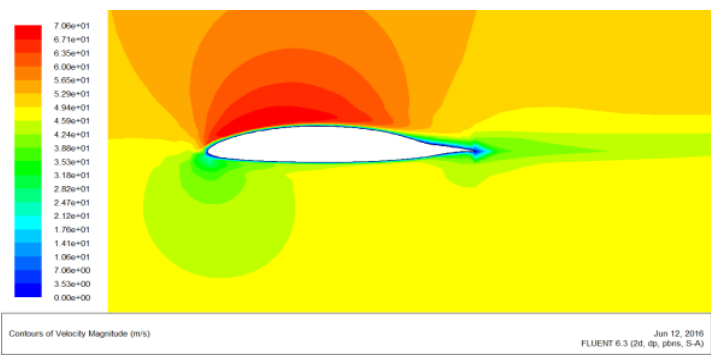

AH79K143

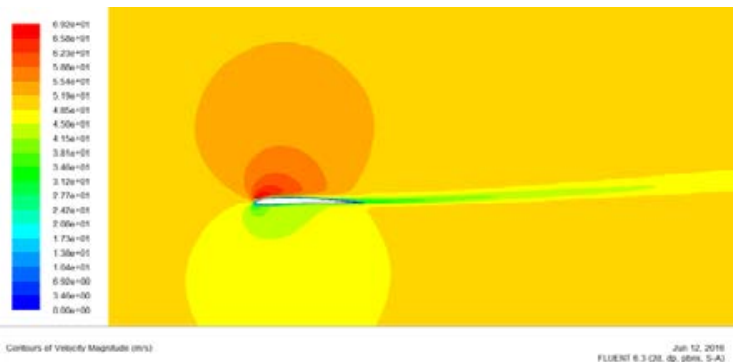

AG19

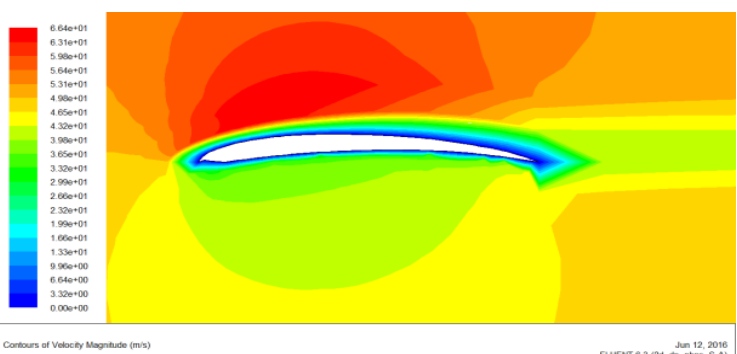

AH7476

FIGURE 5. Distribution of velocity in different profile shapes

\section{Result Analysis}

From numerical simulation result figures, the overall characteristic of air flow for uniform profile is that profile incident upper end nagative pressure is quiet high, and the velocity is lager; profile incident inferior positive pressure is also quiet high, but the velocity is slower. The more closed to the 
edge of profile, the more it is obvious. It also has shock waves in the area. In the wake of propell the fluid velocity is lower.

In this paper, we come to a conclude through contrasting and observing the pressure contour plots and velocity vector(Reynolds number is $3.33 \times 10^{6}$ ) with different angle of attacks and different profile shapes, we draw these conclusions:

a) With the increasing of angle of attacks, the wake of propell is being lager. The changes of boundary layer thickness failed to reflect because of scattered grids.

b) The site presents shock waves on the upper surface of the airfoil continually advanced. When the angle of attacks reach up to $10^{\circ}$, trailing above comes up the similar vortex street stucture. There are closed currents,that is,so called separation vortex. ${ }^{[3]}$ Because rotated vortex pressure is constant, which equals to airflow pressure above, the pressure differential between top and bottom profile surface will reduce a lot. The lift of the wing compared with the original one is decreased. The corresponding angle of attack called "stalling angle of attack "[4]

c) As for airfoil profile shapes, if the airfoil profile upper incident flow surface wider, the negative and high speed pressure distinction is larger; if the airfoil profile bottom more uneven, the positive and low speed pressure distinction is also larger. However, the shock wave phenomenology is less obvious. If the airfoil profile bottom is more horizontal, the wake distinction is greater and more evident. At the same time, the larger incident flow surface, the higher the lift and drag will be.

\section{References}

[1] Zhi-Rong Han. Numerical analysis of the effect of Reynolds number on NACA0012 airfoil flow [J]. China Science information. 2015(15):52-54.

[2] Ye Jian,Guo-Hua Lin,Zheng-Ping Zou,Li-Peng Lu.The Analysis of Flow Around a 2-D Airfoil at Low Reynolds Number [J]. Journal of Aerospace Power. 2003, 18(1):38-45.

[3] Cong-Shan Zhuo,Cheng-Wen Zhong,Li Kai,Jian-Fei Jie,Zhang Yong,Simulation of High Reynolds Number Flow Around Airfoil by Lattice Boltzmann Method [J]. Acta Aeronautica ET Astronautica Sinica,2010, 31(2):238-243.

[4] Song-Ling Wang.Hydrodynamics [M]. China power press,2004. 